\title{
The Turmell-Meter: In Vivo Ankle Kinematics by Using Draw-Wire and Inertial Sensors
}

\author{
Ángel Valera, Óscar Agudelo, and Julio H. Vargas-Riaño*, Member,
}

\begin{abstract}
Objective: To implement a prototype specific for human ankle kinematics studies in limited spaces, immobile, or lying down patients. Based on anatomy and anthropometry, using a screw theory model, draw-wire and inertial sensors were employed. Methods: We included ankle injury studies to highlight the importance of measuring the in vivo range of motion; we studied the ankle anatomy, biomechanics, and anthropometry to estimate the size and movements of the device. We simulated the biaxial representation of ankle motion through the product of exponential mapping. Finally, we designed a structure based on trilateration by projecting tetrahedrons, an acquisition circuit with firmware and calibration software. Results: The prototype has two main parts: support and adjustable platform. We proposed a method to find the position by projecting three apexes on the base using draw-wire sensors, an acquisition board, a single-board computer, a display, Bluetooth, Wi-Fi, and two inertial measurement units. The power source had battery backup with boost and buck converters. Conclusion: We proposed an ankle model in the screw theory framework, a method for localization, and a novel device for in vivo measurements specific for lying patients on a bed, the ground, outdoors, or remote locations without complex setups. The double-battery management is robust and long lasting. Significance: The device is an alternative for measuring the range of motion in laying down patients. We will use it in modeling, diagnosis, and rehabilitation.
\end{abstract}

Index Terms - anthropometry, biomechanics, coordinate measuring machines, in vivo, kinematics, mechanical sensors, sensor arrays, operational amplifiers, pose estimation, position measurement, rehabilitation robotics, biomedical informatics, screw theory

\section{INTRODUCTION}

TURMELL-METER is a hyphenated word from the Valencian language, and its meaning has two components: "ankle" and "measure". In this work, we present a mechatronic application to study the human ankle.

Human ankle modeling and measuring is important in physiology, biomechanics, and robotics for rehabilitation (also in the design of truly humanoid robotic legs). The ankle is a fundamental joint of the human locomotion system and the most commonly reported lower-limb injury in schools, sports, and military activities [1]-[6]. Similar to other human characteristics, the human ankle model has a footprint to identify each human. The variations in individual ankle characteristics are based on anthropometric measurements that depend on sex, age, and phenotype. There is little electronic

$\square$ Submitted August 2021 This work is supported by ColfuturoColciencias.

Ángel Valera is on Ai2 at the Universitat Politècnica de València (email: giuprog@isa.upv.es). equipment specialized for in vivo patient-specific measurement of the ankle in reduced spaces, especially for laying-down patients in remote places.

3D-printed biomedical devices can be personalized, enhanced, scaled, and modified for a specific application. In this work, we designed 3D printed parts for a group of standard sizes.

The screw theory representation of spatial transformations is broadly used in modern robotics. A requirement of the product of the exponential formula is to know the initial pose of a body; it can be referenced to another rigid body by using draw-wire sensors. We employ draw-wire sensors and trilateration to find the initial pose.

Inertial sensors are broadly used for tracking; they give us real-time digital information about the movement, but they have drift. Therefore, we complement the inertial sensor information with draw-wire sensors.

The turmell-meter (TM) should be used for ankle kinematics to compare ankle symmetry or for model validation.

For a rapid visual introduction, we show the device in a typical patient position in Fig. 1.

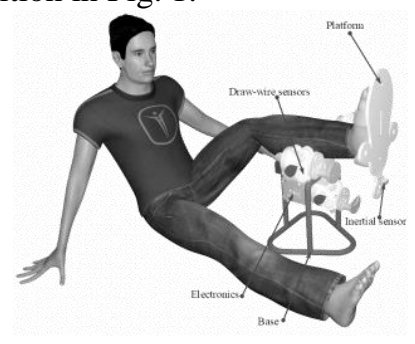

Fig. 1. TM concept in a typical position.

We employ the knowledge and conventions of ankle anatomy and biomechanics to represent an ankle model in the screw theory framework and as a basis to design a prototype to study human ankle kinematics. We presented in brief the concept in [7].

\section{RELATED WORK}

Ankle modeling is part of the screw theory framework, which was introduced by Ball [8], presented in books [9]-[17], applied to multibody systems, and geometrically represented in [18][26]. Modern robotics have used the product of exponential formulas in rigid body motions [27].

Óscar Agudelo is in the Universidad de los Llanos (e- mail: oscar.agudelo@unillanos.edu.co).

Julio H. Vargas-Riaño is a PhD student at the Universitat Politècnica de València (e-mail: julio_h_vargas_r@ieee.org). 
TABLE I MEAN VALUES OF THE ANTRHROPOMETRIC MEASUREMENTS

\begin{tabular}{lllllll}
\hline \hline Values & $\mathrm{K}(\mathrm{cm})$ & $\mathrm{L}(\mathrm{cm})$ & $\mathrm{O}(\mathrm{cm})$ & $\mathrm{P}(\mathrm{cm})$ & $\mathrm{Q}(\mathrm{cm})$ & $R=\frac{W}{w}$ \\
\hline \hline Mean & 1.2 & 1.1 & 1.6 & 1.0 & 0.5 & 0.54 \\
\hline
\end{tabular}

Mean values of $\mathrm{K}, \mathrm{L}, \mathrm{O}, \mathrm{P}, \mathrm{Q}$, and $R=\frac{W}{w}$.

Screw theory has been employed in biomechanics for human jaw characterization [28], [29] and characterization of instantaneous screws in a human knee [30]-[32]. Additionally, inertial measurement units have been applied in tracking limbs by referencing multiple sensors in [33]-[39].

There are different 3D ankle models in the literature; in our work, we focus on the two-axis approach, which is included in the International Biomechanics Society recommendations [40], anatomy and biomechanics books [41]-[45], and simulation software [46]. Specific works on ankle biomechanics are in [47]-[52] and more recently in [53].

In vivo studies for articulated boots were performed in [54], subject-specific in [55], and the axes of rotation were calculated in [56]. Dual fluoroscopy for the ankles from markers was performed in [57].

The most complex joint is the subtalar axis, and important contributions are shown in [58]-[65]. Functional representations in the literature are presented in [66].

Although the TM was inspired by the two-axis model, it can be used in the study and characterization of this and other 3D joint mechanisms, as shown in [67], [68].

Draw-wire sensors have been employed in robotics [69]-[71], linear position tracking [72], and easy robot programming [73]. Inertial measurement units were used by post-processing data and complementing other sensors in [74]-[78].

\section{IIIANKLE BIOMECHANICS}

In this section, we start from the ankle description, which presents a complex movement. First, we study the shank, ankle and foot bones. Then, we analyze the ankle movements based on the anatomic spatial and functional representation.

\section{III.A Ankle Bones}

It is a good idea to start by understanding the morphology of the bones when studying ankle movements.

Fig. 2 identifies the names of the bones of the left and right feet.

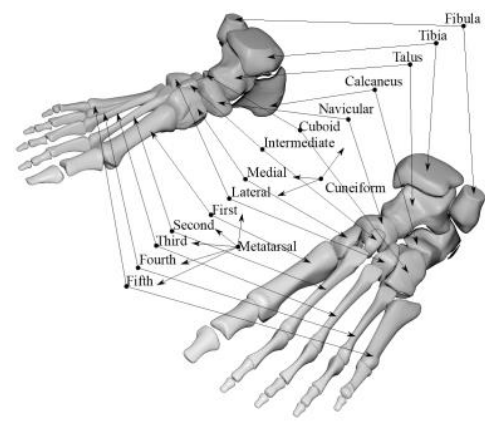

Fig. 2. Foot and ankle bones.

In Fig. 3, we use the right-hand rotation convention and systematically present the movements. Additionally, we organize the movements into two rows, which correspond to pronation and supination. We also show the hindfoot and midfoot as the most involved segments in ankle movements.

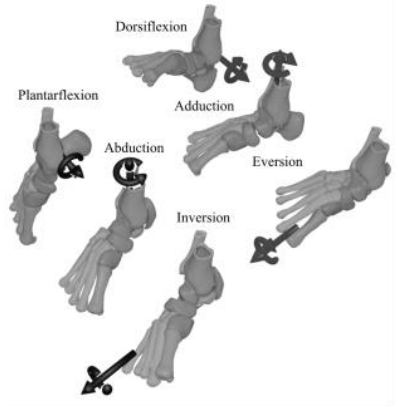

Fig. 3. Ankle movements.

\section{III.B Ankle Kinematic Model}

As we mentioned, the most accepted approach to model the ankle is biaxial movement. As shown in Fig. 3, the ankle movement is the result of the interaction of several bones, such as the fibula, tibia, talus, calcaneus, navicular, cuboid, and three cuneiform bones. However, the mathematical model of the ankle is reduced to a representation of two hinge joints in series, as presented in Fig. 4.

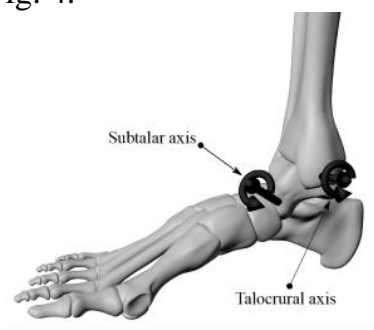

Fig. 4. Two-axis ankle model.

The first axis is related to the tibiofemoral and talus joint and is known as the talocrural (TC) axis; some sources name the joint of the tibiofemoral group and talus dome "mortise" and "tenon" because of the similarity with the architectonic and carpentry structure. The second axis is the subtalar (ST) joint. The bones involved in this rotation are the talus, calcaneus, navicular, and cuneiform groups. To identify those axes, it is necessary to consider the reference frames from each bone. As we show, there are mechanical parallel chains joined by nontrivial surfaces in the ankle structure. It is difficult to localize the reference frame of human bones. Normally, this process is performed using a goniometer, palpation, markers, medical imaging, inertial sensors, or other indirect methods.

\section{ANKLE MOdEl Simulation}

In this section, we extract the data from [79] and scale the model with the proportions from [80], [81] and statistics in [82], [83].

\section{IV.A Reference Points Assignation}

Based on [79], we show in Fig. 5 the reference points and values $\mathrm{K}, \mathrm{L}, \mathrm{O}$ and $\mathrm{P}$. 


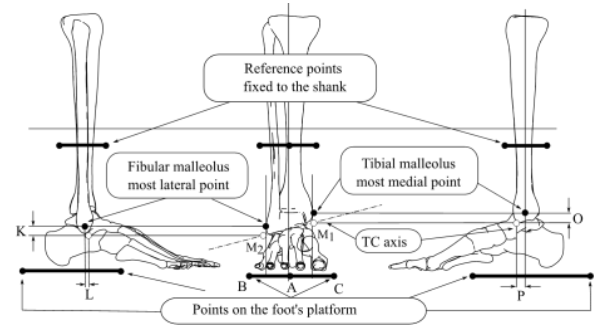

Fig. 5. Reference points from anthropometric values $\mathrm{K}, \mathrm{L}, \mathrm{O}$ and $\mathrm{P}$.

$\mathrm{A}, \mathrm{B}$, and $\mathrm{C}$ are the vertices of a triangle fixed to the foot. Distances K, L, and $\mathrm{O}$ are measured from the most medial and lateral points from the black-filled marker to the white-filled marker. Points $\mathrm{M}_{1}$ and $\mathrm{M}_{2}$ pertain to the $\mathrm{TC}$ axis.

In Fig. 6, the transverse top and right lateral views with distances $\mathrm{Q}, \mathrm{W}$, and $\mathrm{w}$ are identified because points $\mathrm{N}_{1}$ and $\mathrm{N}_{2}$ define the ST axis.

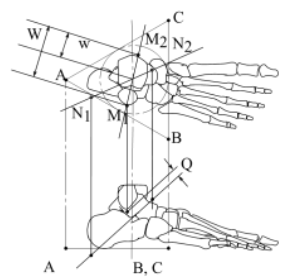

Fig. 6. Transversal and lateral views for values $\mathrm{Q}, \mathrm{W}$ and $\mathrm{w}$.

The mean values in Fig. 5 and Fig. 6 are listed in Table I.

In Fig. 7, we show the ST and TC axes from several viewpoints. The TC axis is measured from the sagittal plane, and the ST is measured from the transverse plane.

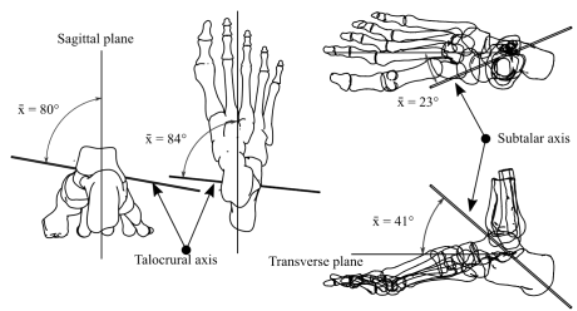

Fig. 7. Mean values of the TC and ST axes.

\section{IV.B Anatomical and geometrical frames Assignation}

We hypothesize that the ankle can be geometrically represented by a 2-dimensional object bounded by a curve, which defines the range of motion (RoM) limits. First, we define the anatomical and geometrical planes as follows: the sagittal (lateral) plane is the $\mathrm{X}-\mathrm{Z}$ plane (perpendicular to the $\mathrm{y}$ axis), the coronal (frontal) plane is the $\mathrm{Y}-\mathrm{Z}$ plane (perpendicular to the $\mathrm{X}$-axis), and the transverse (axial) plane is the $\mathrm{X}$-Y plane (perpendicular to the $\mathrm{Z}$-axis). This correspondence is shown in Fig. 8, left.

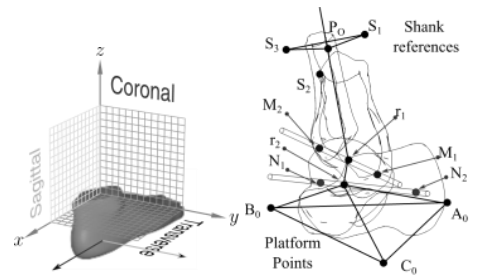

Fig. 8. 3D representation of the planes, bones axes, and reference points.
With this reference frame, we can define the orientation of the TC axis from a unitary vector in the z-direction. We first rotate $-80^{\circ}$ around the $x$-axis and subsequently rotate $-6^{\circ}$ around the z-axis. Similarly, the ST axis can be defined from a unitary vector in the direction of the $\mathrm{x}$-axis by rotating $41^{\circ}$ about the $\mathrm{y}$ axis, followed by a rotation of $23^{\circ}$ around the z-axis.

We summarize the $3 \mathrm{D}$ positions of the fibula, tibia, talus, calcaneus, reference points, $\mathrm{TC}$ and $\mathrm{ST}$ axes in Fig. 8, right. In this image, $\mathrm{A}_{0}, \mathrm{~B}_{0}$, and $\mathrm{C}_{0}$ are the vertices from the platform fixed to the foot. $S_{1}, S_{2}$, and $S_{3}$ are fixed to the shank relative to the arbitrary origin point $\mathrm{P}_{\mathrm{O}} . \mathrm{M}_{1}$ and $\mathrm{M}_{2}$ define the TC axis; $\mathrm{N}_{1}$ and $\mathrm{N}_{2}$ correspond to the $\mathrm{ST}$ axis. We define $\mathrm{r}_{1}$ and $\mathrm{r}_{2}$ as the sagittal plane intersection with the TC and ST axes.

\section{IV.C Size and Dimensions}

After the plane and point assignment, we estimate the device dimensions from anthropometric proportions in [80] and use the segment proportions in Fig. 9.

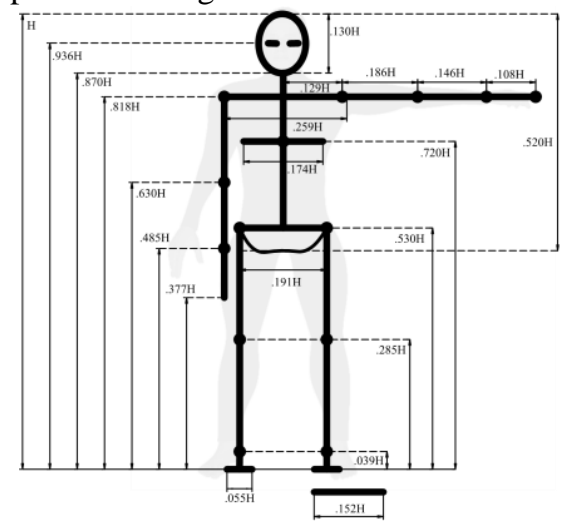

Fig. 9. Body segment proportions.

In our model, the origin is located at the center of the distance between the knee and the ankle. This distance is proportional to $0.246 \mathrm{H}$ of the body height. The distance from the projection of $\mathrm{P}_{\mathrm{L}}$ on the sagittal plane and $\mathrm{P}_{\mathrm{O}}$ is $\mathrm{d}_{\mathrm{m}}$.

$d_{m}=\left\|P_{O}-P_{M}\right\|=\frac{0.246 H}{2}$

According to [82], the mean height $\mathrm{H}$ of an adult male is 175 $\mathrm{cm}$; by substituting this value into the proportion, we have a knee-ankle distance of $21 \mathrm{~cm}$. The distance between points $r_{1}$ and $r_{2}$ about the TC and ST axes on the sagittal plane is:

$d_{p 12}=\left\|r_{1}-r_{2}\right\|=\mathrm{Q}$

The projection of the most medial point on the sagittal plane is

$P_{M}=\left(x_{M}, 0, z_{M}\right)$

and the projection of the most lateral point on the sagittal plane is

$P_{L}=\left(x_{L}, 0, z_{L}\right)$

Point $\mathrm{M}_{1 \mathrm{P}}$ is the projection of $\mathrm{M}_{1}$ on the sagittal plane and calculated from the $\mathrm{P}$ and $\mathrm{O}$ values. $\mathrm{M}_{2 \mathrm{P}}$ is the projection of $\mathrm{M}_{2}$ on the sagittal plane and calculated from the $\mathrm{L}$ and $\mathrm{K}$ values.

$M_{1 P}=\left(x_{M}-\mathrm{P}, 0, z_{M}-0\right)$

$M_{2 P}=\left(x_{L}-L, 0, z_{L}-\mathrm{K}\right)$

The projection of $\overline{M_{2} M_{1}}$ on the sagittal plane is $\overline{M_{2 P} M_{1 P}}$; it has the proportional relation $\mathrm{W} / \mathrm{W}$ with respect to $\overline{M_{2 P} r_{1}}$. Then, $\frac{M_{2}-M_{1}}{M_{2}-r_{1}}=\frac{W}{w}=\mathrm{R}$ 
Solving for $\mathrm{r}_{1}$ gives the following:

$r_{1}=M_{2}-\frac{M_{2}-M_{1}}{R}$

By knowing the distance $Q$ projected in the sagittal plane and $r_{1}$ at an angle of $41^{\circ}$, we calculate $r_{2}$ from

$r_{2}=\mathrm{Q}\left(\cos \left(41^{\circ}\right), 0,-\sin \left(41^{\circ}\right)\right)+r_{1}$

The distance $d_{p}$ from the ankle to the foot is

$d_{p}=0.039 H$

With reference to a circumscribed equilateral triangle with radius $r_{P}$ projected from $r_{1}$ to the platform, the initial distance $d_{z}$ from the origin is

$d_{z}=d_{p}+d_{m}$

The initial points of the platform $\mathrm{A}_{0}, \mathrm{~B}_{0}$, and $\mathrm{C}_{0}$ are

$A_{0}=\left(r_{p}, 0,-d_{z}\right)$

$B_{0}=\left(r_{p} \cos \left(\frac{2}{3} \pi\right), r_{p} \sin \left(\frac{2}{3} \pi\right),-d_{z}\right)$

$A_{0}=\left(r_{p} \cos \left(-\frac{2}{3} \pi\right), r_{p} \sin \left(-\frac{2}{3} \pi\right),-d_{z}\right)$

\section{IV.DProduct of Exponential Mapping}

In this part, we simulate the ankle kinematics using the product of exponential mapping. Following the intuitive concept that the bone surfaces constrain the movement of the ankle, it can be represented as a special Euclidean group of rigid movements $\mathrm{SE}(3)$ of the foot in matrix form

$g_{T}=\left[\begin{array}{cc}\mathbf{R} & p_{T} \\ \mathbf{0} & 1\end{array}\right]$

where $R_{3 \times 3}$ is the rotation matrix, and $\mathrm{p}_{\mathrm{T}}$ is the translation vector.

The initial transformations for points $\mathrm{A}_{0}, \mathrm{~B}_{0}$, and $\mathrm{C}_{0}$ are:

$g_{A}(0)=\left[\begin{array}{cc}\mathbf{I}_{3 \times 3} & A_{0} \\ \mathbf{0}_{\mathbf{1} \times \mathbf{3}} & 1\end{array}\right]$

$g_{B}(0)=\left[\begin{array}{cc}\mathbf{I}_{3 \times 3} & B_{0} \\ \mathbf{0}_{\mathbf{1} \times \mathbf{3}} & 1\end{array}\right]$

$g_{C}(0)=\left[\begin{array}{cc}\mathbf{I}_{3 \times 3} & C_{0} \\ \mathbf{0}_{1 \times 3} & 1\end{array}\right]$

The ends of $\boldsymbol{r}_{1}$ and $\boldsymbol{r}_{\mathbf{2}}$ are the origins of $\boldsymbol{\omega}_{1}$ and $\boldsymbol{\omega}_{2}$. The components $\boldsymbol{v}_{1}=-\boldsymbol{\omega}_{1} \times \boldsymbol{r}_{1}$ and $\boldsymbol{v}_{2}=-\boldsymbol{\omega}_{2} \times \boldsymbol{r}_{2}$ compound the 6-dimensional vectors $\xi_{1}$ and $\xi_{2}$.

$\xi_{i}=\left(\begin{array}{c}\boldsymbol{v}_{\boldsymbol{i}} \\ \boldsymbol{\omega}_{\boldsymbol{i}}\end{array}\right), i=1,2$

where $\boldsymbol{\omega}_{1}$ and $\boldsymbol{\omega}_{2}$ are

$\omega_{1}=\frac{M_{2}-M_{1}}{\left\|M_{2}-M_{1}\right\|}$

$\omega_{2}=\frac{N_{2}-N_{1}}{\left\|N_{2}-N_{1}\right\|}$

The rotation matrix is obtained by the skew-symmetric matrix

$$
\widehat{\omega_{\iota}}=\left[\begin{array}{ccc}
0 & -\omega_{z i} & \omega_{y i} \\
\omega_{z i} & 0 & -\omega_{x i} \\
-\omega_{y i} & \omega_{x i} & 0
\end{array}\right]
$$

With Rodrigues' formula

$e^{\widehat{\omega}_{i} \theta_{i}}=I_{3 \times 3}+\widehat{\omega}_{i} \sin \theta_{i}+\widehat{\omega}_{i}^{2}\left(1-\cos \theta_{i}\right)$

The twist is

$\tau_{i}=\left(\mathbf{I}_{3 \times 3}-\boldsymbol{e}^{\widehat{\omega}_{i} \theta_{i}}\right) \boldsymbol{\omega}_{i} \times v_{i}+\omega_{i} \omega^{T} v_{i} \theta_{i}$
For each joint, $\mathrm{i}=1,2$

$e^{\xi_{i} \theta_{i}}=\left[\begin{array}{cc}e^{\widehat{\omega}_{i} \theta_{i}} & p_{i} \\ \mathbf{0}_{1 \times 3} & 1\end{array}\right]$

Points A, B, and C have invariant relative positions, and there are two rotating joints; the product of the exponential formula for each point is

$\boldsymbol{g}_{\boldsymbol{A}}=e^{\xi_{1} \theta_{1}} e^{\xi_{2} \theta_{2}} g_{A}(0)=\left[\begin{array}{cc}\mathbf{R} & p_{A} \\ \mathbf{0} & 1\end{array}\right]$
$\boldsymbol{g}_{\boldsymbol{B}}=e^{\xi_{1} \theta_{1}} e^{\xi_{2} \theta_{2}} g_{B}(0)=\left[\begin{array}{cc}\mathbf{R} & p_{B} \\ \mathbf{0} & 1\end{array}\right]$
$\boldsymbol{g}_{C}=e^{\xi_{1} \theta_{1}} e^{\xi_{2} \theta_{2}} g_{C}(0)=\left[\begin{array}{cc}\mathbf{R} & p_{C} \\ \mathbf{0} & 1\end{array}\right]$

where $\theta_{1}$ and $\theta_{2}$ are the values of the talocrural and subtalar axes of rotation.

\section{IV.E Code Implementation}

We implement the code in Sagemath. The complete code is in [84]. We show the simulation flexibility by initializing the anthropometrical values in listing 1:

\#Anthropometric values
$\mathrm{vK}=12 ; \mathrm{vL}=11 ; \mathrm{vO}=16 ; \mathrm{vP}=1 ; \mathrm{vQ}=5 ; \mathrm{vR}=0.54$ \#anthropometric measurements
$\mathrm{H}=1750$ \#heigth in mm
d_m=0.246*H \#knee ankle distance
d_p=0.039*H \#ankle sole of the foot distance
z_p=-(dm+d_p) \# z initial position
$r \_p=60$ \#platform radius
ae=2*pi/3 \#auxiliar angle
O=vector $([0,0, \mathrm{~d} m / 2])$ \#middle shank reference point

Listing 1. Sagemath code for the variable initialization.

Additionally, we show the product of exponential implementation in listing 2.

\begin{tabular}{|c|}
\hline 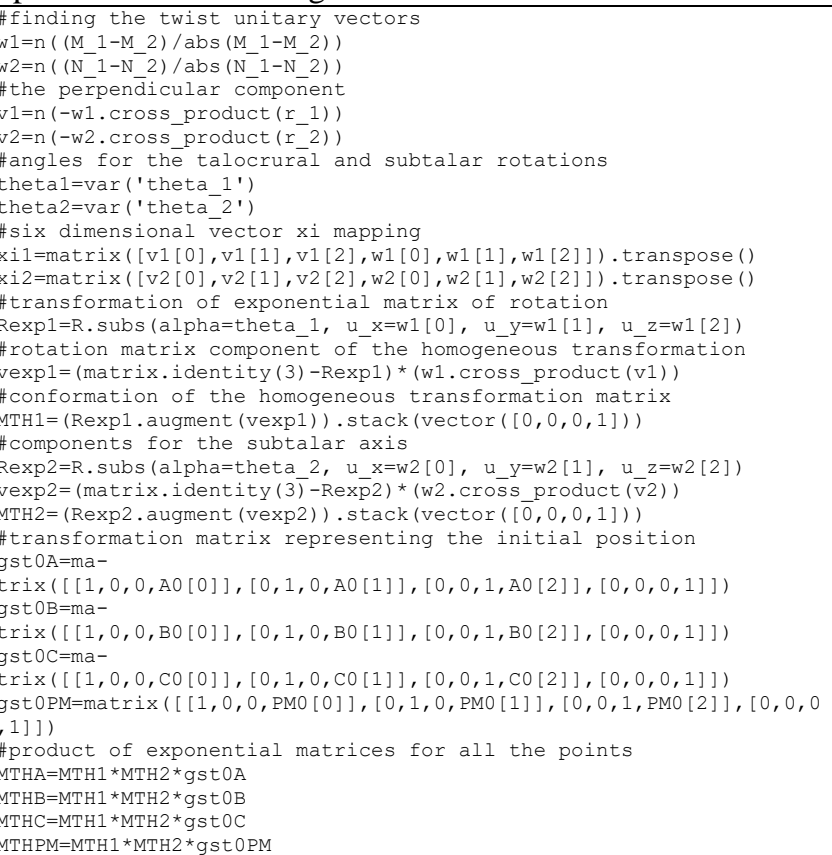 \\
\hline
\end{tabular}

Listing 2. Sagemath code for the product of exponentials.

The graphical result of the simulation for the central point of the platform is shown in Fig. 10. On the left, points $\mathrm{O}, \mathrm{A}_{0}, \mathrm{~B}_{0}$, $\mathrm{C}_{0}, \mathrm{r}_{1}$, and $\mathrm{r} 2$ represent the group of movements of PM. Such representation is useful for the range of motion of the ankle model in all directions. The groups of movement simulations for $\mathrm{A}, \mathrm{B}$, and $\mathrm{C}$ are shown in Fig. 10, right. 


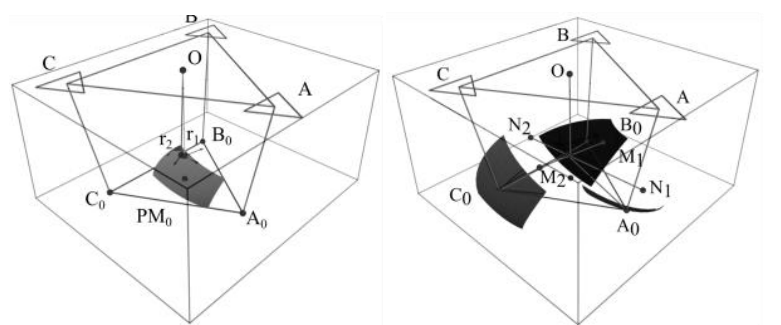

Fig. 10.Movement of the PM and vertices A0, B0 and C0.

As shown in Fig. 10, the trajectories of A, B, C, and PC generated by biaxial movements are smooth surfaces or manifolds. They are mapped by two degrees of freedom with a limited domain due to the range of movement of the axes.

By considering the distances between the origin and the vertices, we estimate the maximal length of the draw-wire sensors in every module.

$l_{m x}=\max \left(\left\|p_{A}\left(\theta_{1}, \theta_{2}\right)-A\right\|+r_{m}\right)$

Here, $l_{m x}$ is the maximal possible length from the triangular inequality, $p_{A}\left(\theta_{1}, \theta_{2}\right)$ is the group of positions in $g_{A}, r_{m}$ is the module radius, and $A$ is the base point.

\section{GEOMETRICAL DESIGN}

The main design requirement is the localization of three points attached to the foot with respect to the shank. We propose to estimate the actual position by using an array of draw-wire sensors in a tetrahedral structure to find the apex location, as shown in Fig. 11.

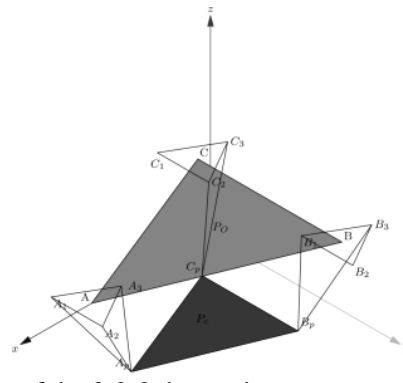

Fig. 11. Design of the 3-2-2 draw-wire sensors

$P_{O}$ and $P_{c}$ are the IMU reference positions. The platform has known dimensions, and the number of sensors is 7. First, we calculate $A_{p}$ from three distances, and $B_{p}$ and $C_{p}$ can be calculated after the first with only two sensors.

In Fig. 11, the tetrahedron $T_{A}$ defined by $A_{1}, A_{2}, A_{3}$, and $A_{p}$ was resolved from the base points and the lengths $l_{A 1}=$ $\left\|A_{1}-A_{P}\right\|, l_{A 2}=\left\|A_{2}-A_{P}\right\|, l_{A 3}=\left\|A_{3}-A_{P}\right\|$.

\section{$V . A$ Finding the $T_{A}$ Apex}

To find $A_{p}$, we realized that the base points are in the same plane as origin $P_{O}$ and developed faces $\Delta_{12 P}\left(A_{1}, A_{2}, A_{P}\right)$, $\Delta_{23 P}\left(A_{2}, A_{3}, A_{P}\right)$, and $\Delta_{13 P}\left(A_{1}, A_{2}, A_{P}\right)$ on the plane of the base, as shown in Fig. 12.

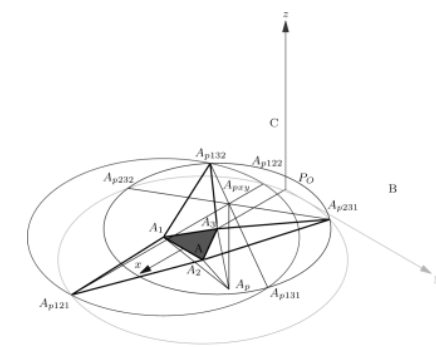

Fig. 12. Tetrahedron A developed on the base plane.

Fig. 12 shows that triangles $A_{1}, A_{3}, A_{p 132}$ and $A_{2}, A_{3}, A_{p 231}$ are two sides of the tetrahedron developed on the base plane.

The respective orthogonal projection of the apex on each adjacent segment of the base triangle can be found by tracing the circle centered on $A_{1}$ with radius $\left\|A_{p}-A_{1}\right\|$ and the circle centered on $A_{3}$ with radius $\left\|A_{p}-A_{3}\right\|$, which results in intersection points $A_{P 132}$ and $A_{p 131}$. In addition, the circle centered at $A_{2}$ with radius $\left\|A_{p}-A_{2}\right\|$ intersects the circle centered at $A_{3}$ at points $A_{p 231}$ and $A_{p 232}$.

The line between points $A_{p 132}$ and $A_{p 131}$ intersects the line defined by points $A_{p 231}$ and $A_{p 232}$ at point $A_{p x y}$. In the case of tetrahedron $T_{A}$, we determined the components $A_{p x}$ and $A_{p y}$ by considering the projection of point $A_{p x y}=\left(A_{p x}, A_{p y}, 0\right)$. It is easy to realize that the height of tetrahedron $T_{A}$ is the distance from the base to point $A_{p}$ and corresponds to the absolute value of the $\mathrm{z}$ coordinate.

If the length of the segments $\left\|A_{1}-A_{2}\right\|,\left\|A_{1}-A_{3}\right\|$ and \| $A_{2}-A_{3} \|$ are the sides of an equilateral triangle, the line defined by points $A_{p}$ and $A_{p x y}$ is perpendicular to the base plane. Then, we can find the distance between points $A_{p x y}$ and $A_{3}$ as the side of a rectangular triangle; the other side is $\mathrm{z}$, and the hypotenuse is the known distance $l_{A 3}=\left\|A_{p}-A_{3}\right\|$.

$A_{z}=\sqrt{l_{A 3}^{2}-\left(A_{p x y}-A_{3}\right)^{2}}$

\section{V.B Tetrahedrons $T_{B}$ and $T_{C}$}

In this stage, by knowing point $A_{p}$, points $C_{p}$ and $B_{p}$ need only two sensors to be found. To determine the result of tetrahedron $T\left(B_{1}, B_{3}, A_{p x y}, B_{P}\right)$, we consider the base as a triangle of known dimensions $B_{1}, B_{2}, \mathrm{~A}_{\mathrm{pxy}}$. We calculated the normal vector perpendicular to the containing plane and calculated the projection similarly to that of tetrahedron $T_{A}$. Considering Fig. 13, only tetrahedron $T_{B}$ is analyzed.

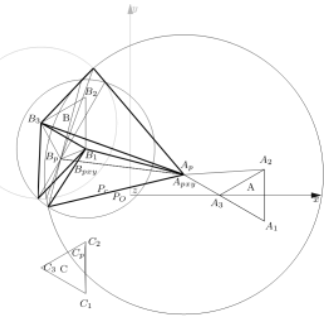

Fig. 13. Resolution of $B_{p x y}$

Finally, to find the projection of the apex of tetrahedron $T_{B}$ 
on the XY plane, we took $B_{p x y}$ as the projection of point $B_{P}$ on the base. The z-coordinate was found by the Pythagorean formula, as shown in the $T_{A}$ case. The same algorithm was applied to tetrahedron $T_{C}$.

\section{V.C Computer Aided Designs}

In this section, we choose draw-wire sensors to measure the lengths of the tetrahedron sides; they are arranged as structural parts. Their maximal length was estimated from the screw simulation. Then, we design the shank attachment and use the shank dimensions, proportions and statistical data to design all other parts.

\section{C.1 Draw-wire Sensor}

We used flat springs with no special characteristics. A detailed sensor study deserves complete publication. The springs are not exposed to a high load against gravity; they are in two or three concurrent groups. Fig. 14 depicts the design, which is composed of three $3 \mathrm{D}=$ printed parts: the potentiometer, flat spring, bolts and nuts.

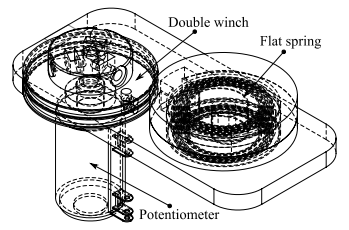

Fig. 14. Draw-wire sensor

A two-coil winch drives the potentiometer; a flat spring retracts a wire attached to the winch. When the wire is extended, the spring retracts it.

The value of each turn was calculated from the nominal value of the potentiometer, $2.2 \mathrm{k} \Omega$, divided into 10 turns, i.e., $220 \Omega$ per turn.

The diameter is $D=3.8 \mathrm{~cm}$, and the spring can be compressed in 4 turns. The maximal length is described as follows:

$l_{\text {max }}=4 \cdot D \cdot \pi$

This value is approximately 477.5 , which is greater than $l_{m x}$ for all groups of movements.

\section{C.2 Mechanical Parts}

The support structure consists of an aluminum tube. The point of attachment on the calf has a size according to the simulation. We used the mesh model of a leg to guide the shape of the calf support. The structure was scaled and divided into 7 parts for 3D printing. A band composed of neoprene and Velcro fabric was attached to the part of the calf.

Fig. 15 shows the mechanical components in the following order: sensor base, foot platform, aluminum structure, and shank support. The TM has 45 3D-printed mechanical parts.

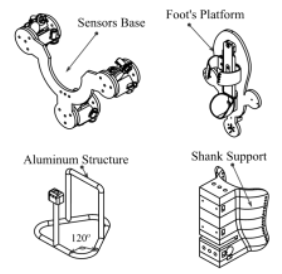

Fig. 15. Mechanical parts.

\section{C.3 Electronics}

The two operational amplifiers configure an instrumentation amplifier as shown in Fig. 16.

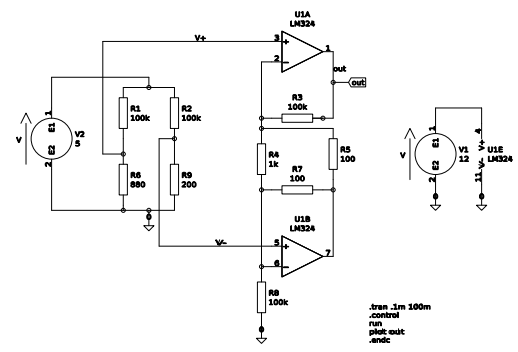

Fig. 16. Instrumentation amplifier.

The voltage gain as follows.

$A_{v}=\frac{v_{o}}{v_{i}}=\left[1+\frac{R_{2}}{R_{1}}+\frac{2 R_{2}}{R_{1}}\right]$

By selecting $R_{2}=100 \mathrm{k} \Omega, R_{1}=1 \mathrm{k} \Omega$, and $R_{G}=5 \mathrm{k} \Omega$,

the gain is 141 .

For $34 \mathrm{mV}$, we obtain:

$v_{o}=v_{i} A_{v}=4.794 \mathrm{~V}$

We designed the printed board circuit as an Arduino ${ }^{\mathrm{TM}} \mathrm{Mega}$ 2560 Shield. All components were incorporated by the throwhole installation.

The device was intended to be portable, so a backup was designed with two packs containing two $18650 \mathrm{Li}$-Ion batteries in series; they have a battery management system, a $5-\mathrm{V}$ buck converter module, and a $12-\mathrm{V}$ boost converter. Fig. 17 shows the schematics.

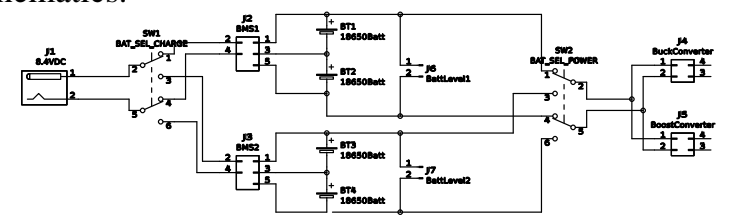

Fig. 17. Backup system.

The output of the batteries can be switched if the voltage level is low. Finally, connectors for the MPUs, OLED and Bluetooth module were added.

\section{C.4 Electronics casing.}

We exported the printed circuit design to Kicad StepUp to fit the case for all components, focusing on a compact configuration.

The two main electronic components are the Arduino Mega 2560 and Orange Pi One. We symmetrically placed components such as the dual pole dual-throw (DPDT) toggle switches on the sides of the box. Fig. 18 shows the main sides and final assembly of the electronic case.

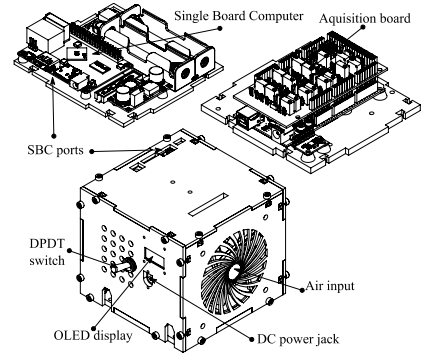

Fig. 18. Electronic Casing 
Every side of the box has attached components to optimize the space and compact the system for portability. Each component can be first calibrated and subsequently installed on the support structure.

\section{C.5 Final mechanical design}

Finally, we design the assembly of all parts, and the main components are attached by an 8-mm steel threaded rod. The subassemblies use M3 bolts and nuts. Fig. 19 shows the assembled design.

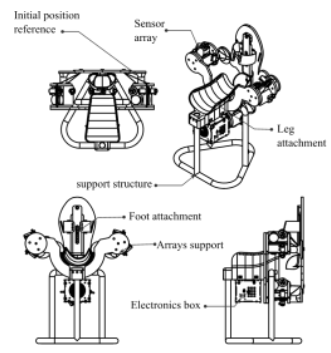

Fig. 19. Final design views.

\section{V.D Calibration and Validation Software}

Calibration was performed with only the Arduino board connected to the PC, which ran a calibration program in Processing. The basic program requests the IMU readings from the accelerometer and gyroscope data and captures the values from the ADC inputs. The raw data are treated as signed integer values 2 bytes wide. The two 1-byte registers were converted to 2-byte integers. An exponentially weighted moving average (EWMA) filter was applied to the raw signals and sent via a serial port to the PC. The lengths were computed from the initial values plus the scaled sensor inputs with

$l_{i M j}=d_{i M j}+\frac{m_{i M j}}{s_{i M j}}$

Here, $l_{i M j}$ is the distance in cm from wire $i$ of module $j, d_{i M j}$ is the initial distance, $m_{i M j}$ is the measured digital value, and $s_{i M j}$ is the scale factor in digital units per $\mathrm{cm}$.

\section{RESULTS}

In this part, we describe the results of the TM design, which are the assembled device and calibration. The CAD are in [85].

First, we show images of the connected electronics parts. Second, the structure was assembled for calibration. Third, the device calibration results are shown. Finally, we placed a healthy leg and foot to show the adaptable ergonomic design of the prototype. We printed the structural parts with ABS, drawwire sensor with PLA and supports and the electronics case with PETG.

\section{VI.A Printed and Connected electronics}

The electronics were assembled in each face of the electronics case. In Fig. 20, the sides were prepared and connected; finally, the assembled case was charged. The revised circuit worked as design simulation and requirements.

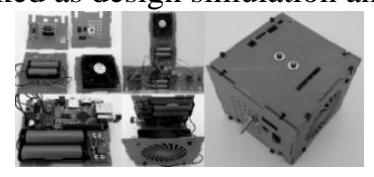

Fig. 20. Connections and electronics

\section{VI.B Printed and Assembled Structure}

All structural components were assembled carefully, and put together with stainless-steel threaded rods. The draw-wire sensors, acquisition board, connections and final structure were independently made for the initial calibration. The resulting image is shown in the figure below.

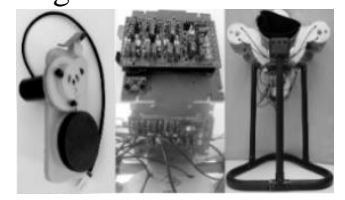

Fig. 21. Assembled Structure.

This system was powered and calibrated with a connected personal computer.

\section{VI.C Calibrated Device}

The resulting calibration was easily performed by using the lengths and a program that captures the signal of the sensors, as shown in Fig. 22.

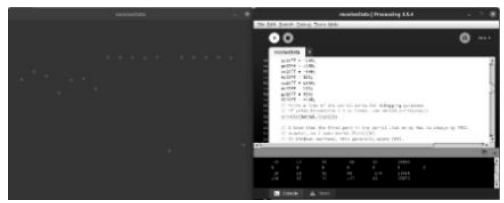

Fig. 22. Processing calibration interface.

The table shows the calibration results.

\begin{tabular}{clllllll}
\hline \hline $\begin{array}{c}\text { Sensor } \\
\text { ID }\end{array}$ & $\mathbf{l 1}$ & $\mathbf{M 1}$ & $\mathbf{1 3}$ & $\mathbf{l 1}$ & $\mathbf{1 2}$ & $\mathbf{l 1}$ & $\mathbf{1 2}$ \\
\hline $\begin{array}{c}\text { BCD value } \\
\text { Measure, } \\
\text { cm }\end{array}$ & 239 & 330 & 246 & 265 & 177 & 252 & $\mathbf{2 4 2}$ \\
\hline \hline
\end{tabular}

\section{VI.DAttached Foot and Shank}

Finally, we show the resulting device attached to a healthy patient. The foot and shank fit in the adjustable platform and support structure, respectively, as is shown in Fig. 23.

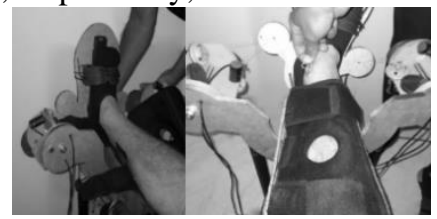

Fig. 23. Attached Foot and Shank.

\section{CONCLUSIONS}

The ankle is the most commonly injured joint of the lower limb; it is important to measure the range of motion by in vivo methods for patients laying down in reduced or remote places. We proposed a device based on the ankle anatomy and anthropometry. Additionally, we used a model in the screw theory framework, which can be characterized by the device. The simulations enabled us to design the size of the device and maximal length of the wires.

We presented a trilateration method by using tetrahedrons projected on the base as an efficient alternative to $3 \mathrm{D}$ sphere intersections.

The draw-wire sensors are modular and a structural part of the device, which is lightweight and portable. The assembly of 
the electronics is also modular, and other single-board computers and microcontroller boards can be used.

The TM will also be used for ankle characterization and diagnosis in the design of a rehabilitation robot named Turmellmoure.

\section{REFERENCES}

[1]A. S. De Boer, T. Schepers, M. J. Panneman, E. F. Van Beeck, and E. M. Van Lieshout, "Health care consumption and costs due to foot and ankle injuries in the Netherlands, 1986-2010," BMC Musculoskelet Disord, vol. 15, p. 128, Apr. 2014, doi: 10.1186/1471-2474-15-128.

[2]M. Waldén, M. Hägglund, J. Orchard, K. Kristenson, and J. Ekstrand, "Regional differences in injury incidence in European professional football," Scandinavian journal of medicine \& science in sports, vol. 23, no. 4, pp. 424430, 2013.

[3]D. M. Swenson, C. L. Collins, S. K. Fields, and R. D. Comstock, "Epidemiology of US High School Sports-Related Ligamentous Ankle Injuries, 2005/06-2010/11:," Clinical Journal of Sport Medicine, vol. 23, no. 3, pp. 190196, May 2013, doi: 10.1097/JSM.0b013e31827d21fe.

[4]D. T.-P. Fong, Y. Hong, L.-K. Chan, P. S.-H. Yung, and K.-M. Chan, "A Systematic Review on Ankle Injury and Ankle Sprain in Sports:," Sports Medicine, vol. 37, no. 1, pp. 73-94, 2007, doi: 10.2165/00007256-20073701000006.

[5] G. D. McKay, P. A. Goldie, W. R. Payne, and B. W. Oakes, "Ankle injuries in basketball: injury rate and risk factors," BrJ Sports Med, vol. 35, no. 2, pp. 103-108, Apr. 2001.

[6]A. Sa, W. Km, S. Ra, and B. Sk, "Epidemiological patterns of musculoskeletal injuries and physical training.," Med Sci Sports Exerc, vol. 31, no. 8, pp. 1176-1182, Aug. 1999, doi: 10.1097/00005768-199908000-00015. [7]J. Vargas-Riaño, Á. Valera, and O. Agudelo Varela, "A Portable Device for Screw Theory Characterization of the Human Ankle," presented at the 19th LACCEI International Multi-Conference for Engineering, Education and Technology, Buenos Aires, Argentina., Jul. 2021. doi: 10.13140/RG.2.2.15112.96001.

[8]R. S. Ball, A Treatise on the Theory of Screws. Cambridge University Press, 1998.

[9]R. Featherstone, Robot Dynamics Algorithms. Springer US, 1987. doi: 10.1007/978-0-387-74315-8

[10] L.-W. Tsai, Robot Analysis: The Mechanics of Serial and Parallel Manipulators. John Wiley \& Sons, 1999.

[11] T. R. Kurfess, Robotics and Automation Handbook. CRC Press, 2004.

[12] B. Siciliano and O. Khatib, Springer Handbook of Robotics. Springer Science \& Business Media, 2008.

[13] X. Kong and C. M. Gosselin, Type Synthesis of Parallel Mechanisms. Springer Berlin Heidelberg, 2010.

[14] J. Angeles, Fundamentals of Robotic Mechanical Systems: Theory, Methods, and Algorithms. Springer Science \& Business Media, 2013.

[15] H. D. Taghirad, Parallel Robots: Mechanics and Control. CRC Press, 2013.

[16] J. Zhao, Advanced theory of constraint and motion analysis for robot mechanisms. Oxford [UK]; Waltham, MA: Academic Press, 2014.

[17] J. Gallardo-Alvarado, Kinematic Analysis of Parallel Manipulators by Algebraic Screw Theory. Springer, 2016.

[18] A. Müller, "Screw and Lie group theory in multibody kinematics," Multibody Syst Dyn, vol. 43, no. 1, pp. 37-70, May 2018, doi: 10.1007/s11044017-9582-7.

[19] E. Minguzzi, "A geometrical introduction to screw theory," Eur. J. Phys., vol. 34, no. 3, pp. 613-632, Mar. 2013, doi: 10.1088/0143-0807/34/3/613.

[20] Z. Terze, A. Mueller, and D. Zlatar, "Lie-Group Integration Method for Constrained Multibody Systems in State Space," Multibody System Dynamics, vol. 33, pp. 1-33, Jul. 2015, doi: 10.1007/s11044-014-9439-2.

[21] J. Pardos-Gotor, Screw Theory for Robotics - A practical approach for modern Robot Mechanics - A compelling computational approach for Screw Theory KINEMATICS. 2018.

[22] J. Solà, J. Deray, and D. Atchuthan, "A micro Lie theory for state estimation in robotics," ArXiv, 2018.

[23] E. Eade, "Lie Groups for 2D and 3D Transformations," p. 25.

[24] Stefano Stramigioli and Herman Bruyninckx, Geometry and Screw Theory for Robotics. ". Accessed: Jun. 20, 2021. [Online]. Available: http://archive.org/details/Stefano_Stramigioli_and_Herman_Bruyninckx_G eometry_and_Screw_Theory_for_Robotics

[25] J. Selig, Geometric Fundamentals of Robotics. 2005.
[26] E. Bayro-Corrochano, Geometric Algebra Applications Vol. II: Robot Modelling and Control. Springer International Publishing, 2020. doi: 10.1007/978-3-030-34978-3.

[27] K. M. Lynch and F. C. Park, Modern robotics: mechanics, planning, and control. University Press, 2017.

[28] J. A. Gal, L. M. Gallo, S. Palla, G. M. Murray, I. J. Klineberg, and C. W. Johnson, "Characterisation of human jaw biomechanics based on screw theory," Australian Journal of Mechanical Engineering, vol. 1, no. 1, pp. 1116, Jan. 2003, doi: 10.1080/14484846.2003.11464460.

[29] J. A. Gal, L. M. Gallo, S. Palla, G. Murray, and I. Klineberg, "Analysis of human mandibular mechanics based on screw theory and in vivo data," Journal of biomechanics, vol. 37, no. 9, pp. 1405-1412, 2004.

[30] S. Martelli and A. Visani, "Computer Investigation Into the Anatomical Location of the Axes of Rotation in the Knee," Journal of Mechanics in Medicine and Biology, vol. 02, no. 03n04, pp. 433-447, Sep. 2002, doi: 10.1142/S0219519402000411.

[31] A. Wolf and A. Degani, "Classifying Knee Pathologies using Instantaneous Screws of the Six Degrees-of-Freedom Knee Motion," in The First IEEE/RAS-EMBS International Conference on Biomedical Robotics and Biomechatronics, 2006. BioRob 2006., Feb. 2006, pp. 1047-1052. doi: 10.1109/BIOROB.2006.1639230.

[32] A. Wolf, "Instantaneous Screws of Weight-Bearing Knee: What Can the Screws Tell Us About the Knee Motion," Journal of Biomechanical Engineering, vol. 136, no. 7, p. 074502, May 2014, doi: 10.1115/1.4027055.

[33] S. Liu, J. Zhang, Y. Zhang, and R. Zhu, "A wearable motion capture device able to detect dynamic motion of human limbs," Nat Commun, vol. 11, no. 1, p. 5615, Nov. 2020, doi: 10.1038/s41467-020-19424-2.

[34] T. McGrath, M. J. Miller, and L. Stirling, "Characterization of Human and Spacesuit Joint Deviations from Body-Worn Inertial Measurement Units," 2021 IEEE Aerospace Conference (50100), 2021, doi: 10.1109/AERO50100.2021.9438204.

[35] R. Krishnan, S. Cruciani, E. Gutierrez-Farewik, N. Björsell, and C. Smith, "Reliably Segmenting Motion Reversals of a Rigid-IMU Cluster Using Screw-Based Invariants," 2018 IEEE-RAS 18th International Conference on Humanoid Robots (Humanoids), 2018, doi: 10.1109/HUMANOIDS.2018.8624969.

[36] A. Ancillao, M. Vochten, E. Aertbeliën, W. Decré, and J. Schutter, "Estimating the Instantaneous Screw Axis and the Screw Axis Invariant Descriptor of Motion by Means of Inertial Sensors: An Experimental Study with a Mechanical Hinge Joint and Comparison to the Optoelectronic System," Sensors, 2020, doi: 10.3390/s20010049.

[37] E. Digo, G. Pierro, S. Pastorelli, and L. Gastaldi, "Tilt-Twist Method Using Inertial Sensors to Assess Spinal Posture During Gait," in Advances in Service and Industrial Robotics, Jun. 2019, pp. 384-392. doi: 10.1007/978-3030-19648-6 44.

[38] K. Parsa, J. Angeles, and A. K. Misra, "Pose-and-twist estimation of a rigid body using accelerometers," in Proceedings 2001 ICRA. IEEE International Conference on Robotics and Automation (Cat. No.01CH37164), May 2001, vol. 3, pp. 2873-2878 vol.3. doi: 10.1109/ROBOT.2001.933057.

[39] A. Barrau and S. Bonnabel, "A Mathematical Framework for IMU Error Propagation with Applications to Preintegration," 2020 IEEE International Conference on Robotics and Automation (ICRA), 2020, doi: 10.1109/ICRA40945.2020.9197492.

[40] G. Wu et al., "ISB recommendation on definitions of joint coordinate system of various joints for the reporting of human joint motion--part I: ankle, hip, and spine. International Society of Biomechanics," J Biomech, vol. 35, no. 4, pp. 543-548, Apr. 2002.

[41] R. A. Mann, "Biomechanics of the Ankle," in Joint Surgery Up to Date, K. Hirohata, M. Kurosaka, and T. D. V. Cooke, Eds. Tokyo: Springer Japan, 1989, pp. 73-81. doi: 10.1007/978-4-431-68096-3_8.

[42] D. A. Winter, Biomechanics and motor control of human movement. John Wiley \& Sons, 2009. Accessed: Jul. 21, 2017. [Online]. Available: https://books.google.es/books?hl=es\&lr=\&id=_bFHL08IWfwC\&oi=fnd\&pg= PA14\&dq=winter+biomechanics++of +the\&ots=JmgrgmaaU4\&sig=OoZBkbS VAcqEddGAGc3F0v2bjgQ

[43] E. J. C. Dawe and J. Davis, "(vi) Anatomy and biomechanics of the foot and ankle," Orthopaedics and Trauma, vol. 25, no. 4, pp. 279-286, Agosto 2011, doi: 10.1016/j.mporth.2011.02.004.

[44] M. J. Coughlin, C. L. Saltzman, and R. A. Mann, Mann's Surgery of the Foot and Ankle E-Book: Expert Consult - Online. Elsevier Health Sciences, 2013.

[45] S. (S. Q. ) Xie, "Kinematic and Computational Model of Human Ankle," in Advanced Robotics for Medical Rehabilitation: Current State of the Art and Recent Advances, S. (S. Q. ) Xie, Ed. Cham: Springer International Publishing, 2016, pp. 185-221. doi: 10.1007/978-3-319-19896-5_7. 
[46] S. L. Delp, J. P. Loan, M. G. Hoy, F. E. Zajac, E. L. Topp, and J. M. Rosen, "An interactive graphics-based model of the lower extremity to study orthopaedic surgical procedures," IEEE Transactions on Biomedical Engineering, vol. 37, no. 8, pp. 757-767, Aug. 1990, doi: 10.1109/10.102791. [47] R. Donatelli, "Normal Biomechanics of the Foot and Ankle," Journal of Orthopaedic \& Sports Physical Therapy, vol. 7, no. 3, pp. 91-95, Nov. 1985, doi: 10.2519/jospt.1985.7.3.91.

[48] J. Dul and G. E. Johnson, "A kinematic model of the human ankle," Journal of Biomedical Engineering, vol. 7, no. 2, pp. 137-143, Abril 1985, doi: 10.1016/0141-5425(85)90043-3.

[49] A. Bähler, "The biomechanics of the foot," Clinical Prosthetics and Orthotics, vol. 10, no. 1, pp. 8-14, 1986.

[50] A. Lundberg, O. K Svensson, G. Németh, and G. Selvik, The axis of rotation of the ankle joint, vol. 71. 1989. doi: 10.1302/0301620X.71B1.2915016.

[51] A. K. Singh, K. D. Starkweather, A. M. Hollister, S. Jatana, and A. G. Lupichuk, "Kinematics of the Ankle: A Hinge Axis Model," Foot \& Ankle International, vol. 13, no. 8, pp. 439-446, Oct. 1992, doi: $10.1177 / 107110079201300802$.

[52] A. Leardini, J. J. O'Connor, F. Catani, and S. Giannini, "A geometric model of the human ankle joint," Journal of Biomechanics, vol. 32, no. 6, pp. 585-591, Jun. 1999, doi: 10.1016/S0021-9290(99)00022-6.

[53] C. L. Brockett and G. J. Chapman, "Biomechanics of the ankle," Orthop Trauma, vol. 30, no. 3, pp. 232-238, Jun. 2016, doi: 10.1016/j.mporth.2016.04.015

[54] D. Bruening and J. Richards, Optimal ankle axis position for articulated boots., vol. 4. 2005.

[55] J. Leitch, J. Stebbins, and A. B. Zavatsky, "Subject-specific axes of the ankle joint complex," Journal of Biomechanics, vol. 43, no. 15, pp. 2923-2928, Nov. 2010, doi: 10.1016/j.jbiomech.2010.07.007.

[56] W. C. H. Parr, H. J. Chatterjee, and C. Soligo, "Calculating the axes of rotation for the subtalar and talocrural joints using 3D bone reconstructions.," Journal of biomechanics, vol. 45, no. 6, pp. 1103-1107, Apr. 2012, doi: 10.1016/j.jbiomech.2012.01.011.

[57] J. A. Nichols, K. E. Roach, N. M. Fiorentino, and A. E. Anderson, "Predicting tibiotalar and subtalar joint angles from skin-marker data with dualfluoroscopy as a reference standard," Gait \& Posture, vol. 49, pp. 136-143, Sep. 2016, doi: 10.1016/j.gaitpost.2016.06.031.

[58] K. A. Kirby, "Subtalar Joint Axis Location and Rotational Equilibrium Theory of Foot Function," Journal of the American Podiatric Medical Association, vol. 91, no. 9, pp. 465-487, Oct. 2001, doi: 10.7547/87507315-91$9-465$.

[59] S. Spooner and K. Kirby, The subtalar joint axis locator: A preliminary report, vol. 96. 2006. doi: 10.7547/0960212.

[60] G. S. Lewis, K. A. Kirby, and S. J. Piazza, "Determination of subtalar joint axis location by restriction of talocrural joint motion," Gait \& Posture, vol. 25, no. 1, pp. 63-69, Jan. 2007, doi: 10.1016/j.gaitpost.2006.01.001.

[61] G. S. Lewis, T. L. Cohen, A. R. Seisler, K. A. Kirby, F. T. Sheehan, and S. J. Piazza, "In vivo tests of an improved method for functional location of the subtalar joint axis," Journal of Biomechanics, vol. 42, no. 2, pp. 146-151, Jan. 2009, doi: 10.1016/j.jbiomech.2008.10.010.

[62] J. De Schepper, K. Van Alsenoy, J. Rijckaert, S. De Mits, T. Lootens, and P. Roosen, "Intratest reliability in determining the subtalar joint axis using the palpation technique described by K. Kirby," Journal of the American Podiatric Medical Association, vol. 102, no. 2, pp. 122-129, 2012.

[63] K. K. Van Alsenoy, J. De Schepper, D. Santos, E. E. Vereecke, and K. D'Août, "The subtalar joint axis palpation technique-part 1: validating a clinical mechanical model," Journal of the American Podiatric Medical Association, vol. 104, no. 3, pp. 238-246, 2014.

[64] K. K. Van Alsenoy, K. D’Août, E. E. Vereecke, J. De Schepper, and D. Santos, "The Subtalar Joint Axis Palpation Technique: Part 2: Reliability and Validity Results Using Cadaver Feet," Journal of the American Podiatric Medical Association, vol. 104, no. 4, pp. 365-374, 2014.

[65] N. Krähenbühl, T. Horn-Lang, B. Hintermann, and M. Knupp, "The subtalar joint: A complex mechanism," EFORT open reviews, vol. 2, no. 7, pp. 309-316, 2017.
[66] J. R. Jastifer and P. A. Gustafson, "The subtalar joint: biomechanics and functional representations in the literature," The Foot, vol. 24, no. 4, pp. $203-$ 209, 2014.

[67] R. D. Gregorio, V. Parenti-Castelli, J. J. O Connor, and A. Leardini, "Mathematical models of passive motion at the human ankle joint by equivalent spatial parallel mechanisms," Medical Biological Engineering Computing, vol. 45, no. 3, pp. 305-313, Mar. 2007, doi: 10.1007/s11517-007-0160-7.

[68] P. Alexandru, Structural-Kinematic Modeling of Human Body Ankle Joint Mechanical Systems - Part I.

[69] J. Andrade-Cetto and F. Thomas, "A Wire-Based Active Tracker," IEEE Transactions on Robotics, vol. 24, no. 3, pp. 642-651, Jun. 2008, doi: 10.1109/TRO.2008.924260.

[70] F. Thomas and L. Ros, "Revisiting trilateration for robot localization," 2005, doi: 10.1109/tro.2004.833793.

[71] F. Thomas, E. Ottaviano, L. Ros, and M. Ceccarelli, "Coordinate-free formulation of a 3-2-1 wire-based tracking device using Cayley-Menger determinants," in 2003 IEEE International Conference on Robotics and Automation (Cat. No.03CH37422), Sep. 2003, vol. 1, pp. 355-361 vol.1. doi: 10.1109/ROBOT.2003.1241621.

[72] M. F. Rahmat, S. Md. Rozali, N. Abdul Wahab, and Z. Has, "Application of draw wire sensor in the tracking control of an electro hydraulic actuator system," 2010, Accessed: Jun. 25, 2021. [Online]. Available: https://core.ac.uk/display/235648634?recSetID=

[73] Z. Jiafan, L. Jinsong, Q. Liwei, and Z. Dandan, "Kinematic analysis of a 6-DOF wire-based tracking device and control strategy for its application in robot easy programming," in 2009 IEEE International Conference on Robotics and Biomimetics (ROBIO), Dec. 2009, pp. 1591-1596. doi: 10.1109/ROBIO.2009.5420394.

[74] A. Bulling, U. Blanke, and B. Schiele, "A Tutorial on Human Activity Recognition Using Body-worn Inertial Sensors," CSUR, vol. 46, no. 3, pp. 133, 2014, doi: 10.1145/2499621.

[75] L. Chermak, N. Aouf, M. A. Richardson, and G. Visentin, "Real-time smart and standalone vision/IMU navigation sensor," 2016, doi: 10.1007/s11554-016-0613-z.

[76] Z. C. Ong and S. Noroozi, "Development of an economic wireless human motion analysis device for quantitative assessment of human body joint," 2018, doi: 10.1016/j.measurement.2017.10.056.

[77] F. Porciuncula et al., "Wearable Movement Sensors for Rehabilitation: A Focused Review of Technological and Clinical Advances," $P M \& R$, vol. 10, no. 9S2, pp. S220-S232, 2018, doi: 10.1016/j.pmrj.2018.06.013.

[78] W. Wahyudi, M. S. Listiyana, S. Sudjadi, and N. Ngatelan, "Tracking Object based on GPS and IMU Sensor," 2018, Accessed: Jun. 25, 2021. [Online]. Available: https://core.ac.uk/display/196524512?recSetID=

[79] R. E. Isman, V. T. Inman, and P. M. Poor, "Anthropometric studies of the human foot and ankle," Bull Prosthet Res, vol. 11, no. 10, pp. 97-129, 1969. [80] R. Drillis, R. Contini, New York University, and School of Engineering and Science, Body segment parameters. New York, N.Y.: New York University, School of Engineering and Science, 1966.

[81] M. Hebbelinck and W. D. Ross, "Kinanthropometry and biomechanics," in Biomechanics IV, Springer, 1974, pp. 535-552. Accessed: Jul. 13, 2017. [Online]. Available: http://link.springer.com/chapter/10.1007/978-1-34902612-8_81

[82] C. D. Fryar, M. D. Carroll, Q. Gu, J. Afful, and C. L. Ogden, "Anthropometric Reference Data for Children and Adults: United States, 20152018," Vital Health Stat 3, no. 36, pp. 1-44, Jan. 2021.

[83] J. Mcconville, C. E. Clauser, T. Churchill, J. Cuzzi, and I. Kaleps, "Anthropometric Relationships of Body and Body Segment Moments of Inertia," 1980. doi: 10.21236/ada097238.

[84] J. H. V. Riaño, TurmellMetre0.1. 2021. Accessed: Aug. 05, 2021. [Online]. Available: https://github.com/juliohvr/TurmellMetre0.1

[85] J. Vargas Riaño, Á. Valera, and O. Agudelo Varela, "Turmell-metre |3D CAD Model Library | GrabCAD," Turmell-metre. https://grabcad.com/library/turmell-metre-1 (accessed Aug. 05, 2021). 